Int. J. Dev. Biol. 53: 1089-1095 (2009)

doi: $10.1387 / \mathrm{ijdb} .072507 \mathrm{sr}$

\title{
Expression of a Prrxl1 alternative splice variant during the development of the mouse nociceptive system
}

\author{
SANDRA REBELO, CLÁUDIA LOPES, DEOLINDA LIMA and CARLOS REGUENGA* \\ Laboratório de Biologia Celular e Molecular, Faculdade de Medicina, IBMC-Instituto de Biologia Molecular \\ e Celular, Universidade do Porto, Portugal
}

\begin{abstract}
Background Gene expression can be differentially regulated by alternatively spliced transcription factors, providing a mechanism for precise control of diverse morphogenetic events. The paired-type homedomain transcription factor Prrxl1 (formerly known as Drg11) was described as a key regulator of the differentiation of the spinal cord neuronal circuit dedicated to the processing of nociceptive information. Here, we report the characterization of a PrrxI1 alternative splice variant that we termed Prrxl1-b. Methods Mouse PrrxI1 isoform mRNA sequences were obtained by Rapid Amplification cDNA Ends (RACE) analysis. The distribution and the amount of Prrxl1-b at different developmental ages were analyzed by in situ hybridization and quantitative real-time PCR, and compared with those of Prrxl1. Results The amount of Prrxl1 was higher than that of the Prrxl1-b isoform both in the DRG and the spinal cord. Prrxl1-b contains the $\mathrm{N}$-terminal homeodomain but differs from the previously identified Prrxl1 in the C-terminal part due to alternative mRNA processing. This results in the lack of the OAR domain in the Prrxl1-b primary structure. PrrxI1-b is exclusively localized in neurons primarily involved in the processing of the pain somatosensory modality. Prrxl1-b presents the same regional distribution pattern as PrrxI1, but differs as to the qualitative and quantitative expression profile at distinct developmental ages in the dorsal root ganglion and spinal cord. Conclusion We suggest that the tissue-specific role of the PrrxI1 gene may be sustained by an accurate balance in the ratio between the amount of PrrxI1 and its OAR-lacking variant, Prrxl1-b, which may be critical during nociceptive circuit development.
\end{abstract}

KEY WORDS: Prrxl1, homeodomain, splice variant, nociception, Drg11

Homeodomain proteins are a large family of transcription factors that have been described as important regulators of morphogenesis events (Gehring, 1992). These proteins are characterized by a conserved 60 aminoacid DNA-binding structure, known as the homeodomain that forms three alpha-helices (Gehring et al., 1994; Dragan et al., 2006). This helix-turn-helix structure binds cooperatively as homo- and heterodimers to palindromic DNA regulator sequences (Wilson et al., 1993). Among the different classes of homeoproteins categorized so far, an important group with major roles in embryonic development is that of paired class proteins, characterized by the presence of an additional 128amino-acid DNA binding domain, referred as the paired domain, located upstream of the homeodomain (Treisman et al., 1991). A related category is the paired-like homeodomain proteins that share high similarity with the paired class proteins but contain a sole DNA-binding region. Additional conserved regions located outside the homeodomain define different subsets of paired-type transcription factors. One of these subfamilies is characterized by the presence of a conserved 14 amino acid motif known as OAR domain, initially described in the homeobox genes Otp (Semina et al., 1996) and Aristaless (Gage and Camper, 1997), located in the carboxyl-terminal tail. To date, the function of this domain is not properly understood. However the exclusive and consistent presence of this motif in the C-terminal of a subset of paired-like homeodomain proteins suggests that it has a molecular function directly related to the transcriptional activity of these factors. One member of this OAR containing paired-type homeodomain family

Abbreviations used in this paper: DRG, dorsal root ganglia; OAR, Otp-AristalessRax domain; ORF, open reading frame; Prrxll, paired related homeobox protein-like 1.

\footnotetext{
*Address correspondence to: Dr. Carlos Reguenga. Instituto de Histologia e Embriologia, Faculdade de Medicina do Porto, Alameda Hernani Monteiro, 4200 319 Porto, Portugal. Tel: +351-22551-3654. Fax: +351-22551-3655. e-mail: cregueng@med.up.pt
} 
is the transcription factor Prrx/1 (official name according to the Mouse Genome Database nomenclature (Eppig et al., 2005)), also referred as Drg11.

Analysis of Prrxl1 mutant mice revealed a critical role of this protein in the development of the spinal cord dorsal horn neuronal circuit dedicated to the processing of nociceptive information. Prr $x / 1^{\%}$ mice exhibit diminished nociceptive behaviour in several pain tests (Chen et al., 2001). Moreover, the differentiation of superficial spinal cord layers is impaired, resulting in structural and neurochemical spinal defects (Chen et al., 2001) and a marked reduction of the number of small primary afferent neurons (Rebelo et al., 2006a).

Here, we report the characterization of a Prrxl1 alternative splice variant that we termed Prrxl1-b. This isoform differs from Prrxl1 in the C-terminal part. The resulting protein contains the $\mathrm{N}$ terminal homeodomain but lacks the C-terminal OAR domain. Moreover, the distribution and the amount of Prrxl1-b at different developmental ages were compared with those of Prrxl1.

\section{Identification of Prrxl1-b and splicing organization of the Prrxl1 gene}

Recently we have characterized the Prrxl1 expression by western-blotting analyses using spinal cord extracts at different developmental ages (Rebelo et al., 2007). Distinct bands were detected suggesting the existence of Prrxl1 isoforms. This observation led us to perform an extensive homology search in GenBank database using the previously known rat Prrxl1 cDNA sequence (accession number NM_145767) (Saito et al., 1995). From this analysis, a murine Prrxl1 related sequence (accession number BC145917) was identified. This cDNA sequence presents an Open Reading Frame (ORF) distinct from that previously described in the rat (Saito et al., 1995), as observed by the nucleotide sequence alignment in Fig. 1A, suggesting the existence of a Prrxl1 variant, which we termed Prrxl1-b. Although the Prrxl1 role has been analyzed in the mouse (Chen et al., 2001; Rebelo et al., 2006a), the murine sequence was not determined so far. We looked for the murine homologous of the previously known rat Prrxl1 using the genomic information of the contig NT-039606. The predicted mouse Prrxl1 ORF sequence was assembled (Fig. 1A). To validate the existence of the two Prrxl1 isoforms, we searched for the respective full-length mRNA. We isolated the 5' and 3' untranslated regions (UTR) by Rapid Amplification cDNA ends (RACE) analyses using primers that hybridize in a region of the open reading frame restricted to each isoform. Only one band corresponding to the 3'UTR of each isoform was obtained (marked by asterisks in Fig. 1B). On the contrary, several sequences with 5 'UTR regions of different size were identified suggesting the presence of multiple Prrxl1 transcription start points in the spinal cord (Bands 1 to 3 in Fig. 1B). The sequence of each transcript is presented in Fig. 1C. Note that band 2 in the Prrxl1 5'-RACE reaction contains two different transcripts of same size. From all the obtained transcripts we selected the transcript containing the longer 5'UTR in order to assemble the cDNA sequences of both isoforms (Fig. 2B). The Prrxl1 and Prrxl1-b nucleotide sequences have been submitted to GenBank and have been assigned the accession number EU670677 and EU670678, respectively. BLAST searches in the mouse EST (Expressed Sequenced Tag) data- base allowed the identification of an incomplete Prrxl1 cDNA sequence (accession number BY729985) derived from DRG extracts, starting at the same nucleotide as those identified in our 5 '-RACE analyses. This observation suggests that the Prrxl1 and Prrxl1-b mRNA sequences identified may correspond to the main transcripts in the diverse types of Prrxl1-expressing neurons.

Based on the information of the contig NT-039606, the alignment between the cDNA of the two isoforms and genomic sequences was performed in order to define the splicing organization of the Prrxl1 gene (Fig. 2A). The murine Prrxl1 gene is located on Chromosome 14 spanning approximately $47 \mathrm{~kb}$ and gave rise, by alternative processing of the last exon, to two transcripts: Prrxl1 (the murine homologous to the previously known rat Prrxl1) and the spliced variant Prrxl1-b. The two mRNAs encode proteins that have the first 175 amino acids in common due to exons 1-6 which encode the $\mathrm{N}$-terminal paired-type homeodomain (Fig. 2C). The C-terminal OAR domain present in Prrxl1 amino acid sequence is missing in Prrxl1-b due to the substitution of the exon 7 by two exons specific to Prrxl1-b sequence (Fig. 2 B,C). Prrxl1b contains 220 aminoacids, making this protein approximately 5 kDa shorter than Prrxl1.

\section{Expression of Prrxl1 isoforms}

The Prrxl1 expression pattern has been previously defined by in situhybridization (Chen et al., 2001; Qian et al., 2002; Ding et al., 2003) and immunohistochemistry (Rebelo et al., 2007). However, the probe and the antibody used in these studies did not discriminate between Prrxl1 and the spliced variant Prrxl1-b. In order to define the regional distribution of Prrxl1-b, as compared to that of Prrxl1, in situ hybridization studies were performed in embryonic day (E) 15.5 and E18.5 embryos using alternatively spliced exon 7-specific riboprobes (Fig. 3). As previously described for Prrxl1 (Chen et al., 2001; Qian et al., 2002; Ding et al., 2003; Rebelo et al., 2007), both Prrxl1 and Prrxl1-b mRNA were expressed in the trigeminal, facial and glossopharyngeal ganglia (Fig. $3 A, C, G, E$ ), the dorsal root ganglia (DRG) (Fig. $3 \mathrm{D}, \mathrm{H}$ ), the trigeminal spinal nucleus (Fig. $3 \mathrm{~B}, \mathrm{~F}$ ) and the spinal cord dorsal horn (Fig. $3 \mathrm{D}, \mathrm{H}$ ). As expected, all the structures expressing Prrxl1-b are involved in the processing of nociceptive information. Although no obvious significant differences in the localization of the two isoforms were detected, there appeared to be a dramatic reduction in the expression of Prrxl1-b in the spinal cord at E18.5, as compared to the expression of Prrxl1 mRNA at the same age. This could be due to the fact that the Prrxl1-b mRNA expressing levels were not high enough to be detected by the riboprobe. No such difference was observed in the DRG.

To further confirm the presence of Prrxl1-b expression in the E18.5 embryo spinal cord, Reverse Transcriptase-PCR analyses were performed using primers that enable the amplification of a sequence specific to each isoform. Total RNA extracts were obtained from various tissues of E18.5 embryos (Fig. 4). As expected both transcripts were only observed in nervous tissues. Limited expression of Prrxl1 was detected in the brain, which may have been due to the use of whole brain extracts since the expression of this transcription factor is restricted to a few regions of the brainstem (Qian et al., 2002; Ding et al., 2003; Rebelo et al., 2007). As to the DRG at this stage of development, both isoforms 
A

Rt Prrx11 (NM_145767) Ms Prrxi1 (assembled) Ms Prrx11-b clone (BC145917)

Rt Prrx11 (NM_145767) MS Prrxi1 (assembled) Ms Prrx11-b clone (BC145917)

Rt Prrx11 (NM_145767) Ms Prrx11 (assembled) Ms Prrx11-b clone (BC145917) RT Prrx11 (NM_145767) MS Prrxl1 (assembled) Ms Prrx11-b clone (BC145917) Rt Prrx11 (NM_145767)
MS Prrx11 (assembled) Ms Prrx11-b clone (BC145917)

Rt Prrx11 (NM_145767) Ms Prrx11 (assembled) Ms Prrx11-b clone (BC145917)

Rt Prrx11 (NM_145767) Ms Prrx71 (assembled)
ATGTTTTATTTCCACTGCCCGCCACAGCTAGAGGGCACAGCGCCTTTTGGTAACCACTCTACGGGGGATTTTGATGATGGGTTTCTTAGAAGAAAACAGCGCAGAAATCGGACAACCTTC 120 ATGTTTTATTTCCACTGTCCGCCACAGCTAGAGGGCACTGCGCCTTTTGGGAACCACTCTACAGGGGATTTTGATGATGGGTTTCTTAGAAGAAAACAGCGCAGAAATCGAACGACCTTC 120 ATGTTTTATTTCCACTGTCCGCCACAGCTAGAGGGCACTGCGCCTTTTGGGAACCACTCTACAGGGGATTTTGATGATGGGTTTCTTAGAAGAAAACAGCGCAGAAATCGAACGACCTTC 120

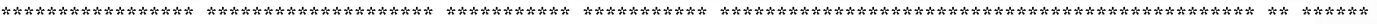
GCTCTTCAGCAGTTGGAAGCTCTGGAGGCAGTCTTTGCCCAAACACACTACCCAGATGTCTTCACCAGAGAAGAGCTAGCCATGAAAATAAACCTCACAGAAGCCAGAGTGCAGGTTTGG 240 ACTCTTCAGCAGCTGGAAGCTCTGGAGGCAGTCTTTGCCCAAACACACTACCCAGATGTCTTCACCAGAGAAGAGTTAGCCATGAAAATAAACCTCACAGAAGCCAGAGTGCAGGTTTGG 240 ACTCTTCAGCAGCTGGAAGCTCTGGAGGCAGTCTTTGCCCAAACACACTACCCAGATGTCTTCACCAGAGAAGAGTTAGCCATGAAAATAAACCTCACAGAAGCCAGAGTGCAGGTTTGG 240

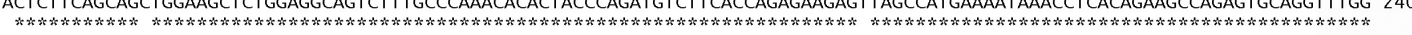
TTCCAGAACCGAAGAGCCAAGTGGAGGAAGACAGAGAGAGGGGCCTCTGACCAGGAACCAGGGGCTAAGGAACCCATGGCAGAGGTGACACCACCCCCAGTGAGGAACATCAACTCTCCA 360 TTCCAGAACCGAAGAGCCAAATGGAGGAAGACAGAGAGAGGGGCCTCTGACCAGGAGCCAGGGGCCAAGGAACCCATGGCAGAGGGTGACACCACCGCCAGTGAGGAACATCAACTCTCCA 360 TTCCAGAACCGAAGAGCCAAATGGAGGAAGACAGAGAGAGGGGCCTCTGACCAGGAGCCAGGGGCAAGGAACCCATGGCAGAGGTGACACCACCGCCAGTGAGGAACATCAACTCTCCA 360 w CCCCCAGGGGACCAGGCCCGGGGCAAGAAGGAGGCCCTGGAGGCCCAGCAGAGCCTGGGACGCACAGTGGGCCCCGCCGGGCCTTTCTTCCCCTCCTGCTTGCCAGGGACCCTCCTGAAC 480 CCCCCAGGGGACCAGACCCGGAGCAAGAAGGAGGCCCTGGAGGCCCAGCAGAGCCTGGGACGCACAGTGGGCCCTACCGGGCCTTTCTTCCCCTCCTGCTTGCCAGGGACCCTCCTGAAC 480 CCCCCAGGGGACCAGACCCGGAGCAAGAAGGAGGCCCTGGAGGCCCAGCAGAGCCTGGGACGCACAGTGGGCCCTACCGGGCCTTTCTTCCCCTCCTGCTTGCCAGGGACCCTCCTGAAC 480 "w* ACAGCCACTTATGCCCAGGCCCTGTCCCATGTGGCATCTCTGAAAGGGGGCCCACTGTGCTCTTGCTGCGTCCCAGACCCTATGGGGCTCTCCTTCCTCCCCACTTACGGTTGCCAGAGT 600 ACAGCCACGTATGCCCAGGCCCTGTCCCATGTGGCATCTICT GA $\overline{A A} \overline{G G} \overline{G G} \overline{G C} \overline{C C} \overline{A C} \overrightarrow{T G T G C T C T T G C T G T G T C C C A G A C C C G A T G G G A C T C T C C T T M C T C C C C A C T T A T G G C T G C C A G A G C}$ 600

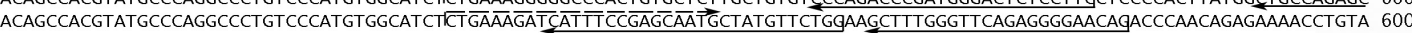

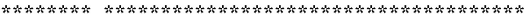

AACCGCACAGCCAGCGTGGCTGCCCTGCGCATGAAGGCCCGCGAGCATTCAGAAGCGGTCCTGCAGTCTGCCAACCTTCTGCCGTCCACCAGCAGCAGCCCCGGCCCTGCCTCCAAGCAG 720 AACCGCACGGCCAGCGTGGCTGCCCTGCGCATGAAGGCCCGCGAGCATTCAGAAGCTGTCCTGCAGTCTGCCAACCTCCTGCCATCCACCAGCAGCAGTCCCGGCCCTGCTTCCAAGCAG 720

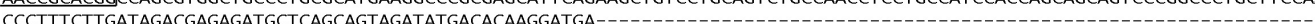

GTGCCTCCAGAAGGCAGCCAGGACAAGCCCTCCCCAACGAAGGAACAGAGCGAGGGAGAGAAGAGCGTATGA 792 GCGCCTCCAGAAGGCAGCCAGGACAAGACCTCCCCAACCAAGGAACAGAGCGAGGGAGAGAAGAGTGTATGA 792

B

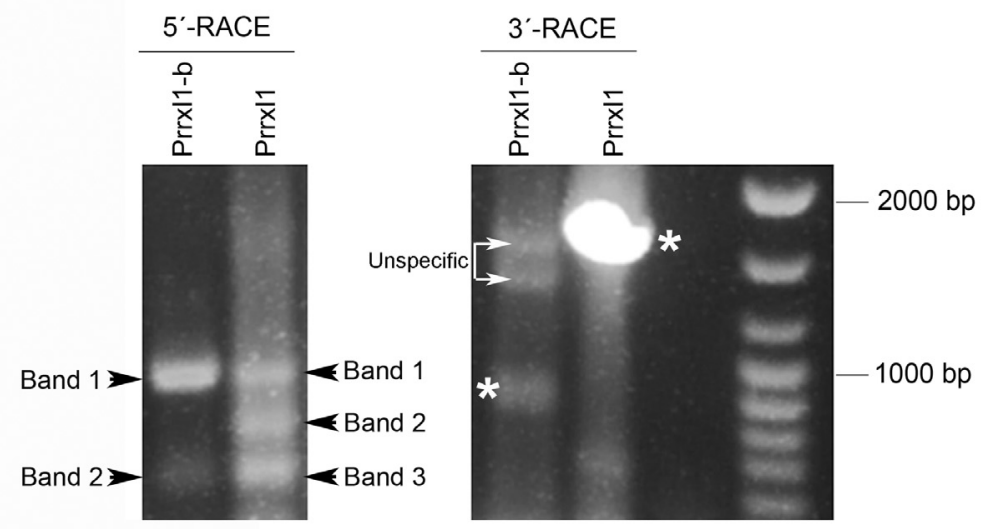

C

\section{$5^{\prime}-\mathrm{RACE}$}

Prrxl1-b

Band 1 aagacatctggccacttccccagtcacttctctggtcctgcgggcgccagcagcgccaagccccgcccgccgccagccgagccccgcgtgcggactagaggcgagcgcactccggccttctcgcccgcctcccgcagagccaggccg Band

Band 1 gagccacgcacaggcacaagtctgccaggccgctaccagccetggtccagccatcagggcccatccgaccgtcggcgATGTTTTAT................AAGATCATTTCCGAGCAATGCTATGTTCTGGAAGC attgctgtttgctttgcagccaggcc Band 2 gagccacgcacaggcacaagtctgccaggecgctaccagccctggtccagccatcagggcccatccgaccgtcggcgATGTTTTAT.......................AGATCATTTCGAGCAATGCTATGTTCTGGAAGC inner primer

Band 1 aagacatctggccacttccccagtcacttctctggtcctgcgggegccagcagcgccaagccccgcccgccgccagccgagccccgcgtgcggactagagg cgagcgcactccggecttctcgcccgcctcccgcagagccaggecg Band 2[ gecgagcccegcgtgcggactagaggegagcgcactccggecttctcgcccgectcccgcagagccaggecg

Prrx|1

Band 1 gagccacgcacaggcacaagtctgccaggccgctaccagccetggtccagccatcagggcccatccgaccgtcggcgATGTTTTAT.....

Band 2 [ gagccacgcacaggcacaagtctgccaggecgctaccagccctggtccagccatcagggcccatccgaccgt cggcgATGTTTTAT.

Band 3 gagccacgcacaggcacaagtctgccaggecgctaccagccctggtccagccatcagggcccatccgaccgt cggcgATGTTTTAT................... TTGTGTCCAGACCCGATGGGACTCTCCTTCCTCC

inner primer

Fig. 1. Identification of Prrxl1 and Prrxl1-b transcripts. (A) Nucleotide sequence alignment of the Open Reading Frame regions corresponding to the previously known rat Prrxl1 (NM_145767), the murine Prrxl1 (assembled by comparison with the genomic data in contig NT-039606) and the murine Prrxl1-b clone (BC145917). The primers used for 5' and 3'-RACE reactions are presented. (B) Gel electrophoresis analysis of the 5' and 3'-RACE reactions. The asterisks and the arrowheads mark the Prrxl1 and Prrxl1-b specific bands in, respectively, the 3' and 5'-RACE reactions. (C) Nucleotide sequences corresponding to the multiple transcripts obtained in the 5'-RACE analysis. The 5'-UTR nucleotides are in lowercase while the ORF nucleotides are in uppercase. 
A

B

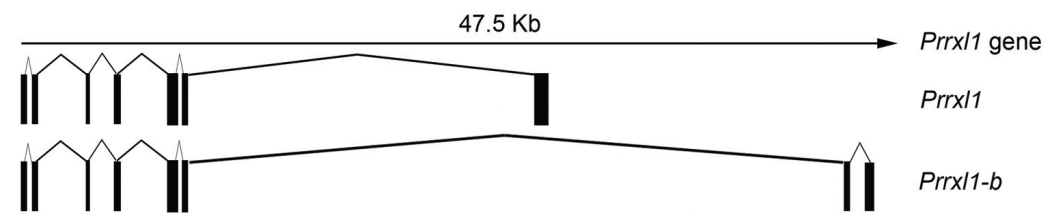

Prrx11

Prrx11-b

Prrx11

Prrx11-b

Prrxi1

Prrx11-b

Prrx11

Prrx11-b

Prrx11

Prrx11-b

Prrx11

Prrx11-b

Prrxi1

Prrx11-b

Prrx11

Prrx11-b

Prrx11

Prrx11-b

Prrx11

Prrx11-b

Prrx11

Prrx11-b

Prrx11

Prrx11-b

Prrx11

Prrx11-b

Prrx11

Prrx11-b

Prrx11

Prrxi1

Prrxi1

Prrx11

Prrxi1

Prrxi1

Prrx11

C

PRRXL1 PRRXL1-B

PRRXL1

PRRXL1-B

MINLTEARVQVWFQNRRAKWRKTERGASDQEPGAKEPMAEVTPPPVRNINSPPPGDQTRSKKEAL 133

MKINLTEARVQVWFQNRRAKWRKTERGASDQEPGAKEPMAEVTPPPVRNINSPPPGDQTRSKKEAL 133

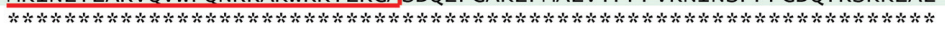

PRRXL1

PRRXL1-B

EAQQSLGRTVGPTGPFFPSCLPGTLLNTATYAQALSHVASLKGGPLCSCCVPDPMGLSFLPTYGCQ 199

EAQQSLGRTVGPTGPFFPSCLPGTLLNTATYAQALSHVASLKDHFRAMLCSGSFGFRGEQTQQRAL 199

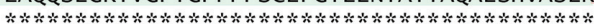

OAR

PRRXL1 SNRTASVAALRMKAREHSEA VLQSANLLPSTSSSPGPASKQAPPEGSQDKTSPTKEQSEGEKSV 263

PRRXL1-B TPNLYPFLIDERCSAVDMTQG-_-
Fig. 2. Characterization of Prrxl1 isoforms. (A) Genomic organization of the murine Prrxl1 gene, and of the Prrxl1-b isoform. Black boxes indicate the exons. (B) mRNA sequence alignment of Prrxl1 and Prrxl1-b. The arrows indicate the boundary between exons. The ORFs are color coded as follows: green indicates identical region between both isoforms, while blue and red indicate specific Prrxl1 and Prrx/1b regions respectively. (C) Amino acid alignment between murine Prrxl1 and Prrx/1$b$ sequences. Identical residues between sequences are marked with an asterisk. The presences of the characteristic homeodomain and the OAR motif specific to the Prrxl1 sequence are highlighted with a box. 
Prrxl1 b
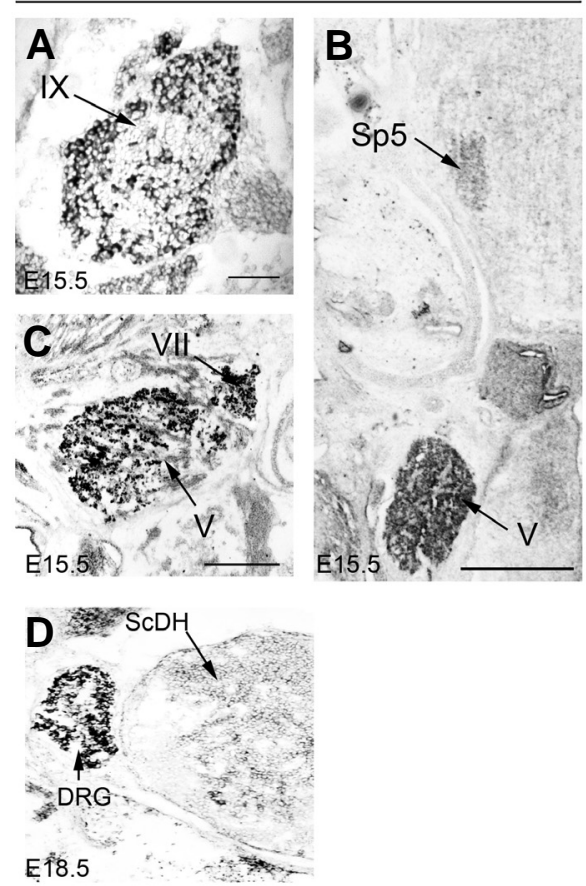

Prrxl1
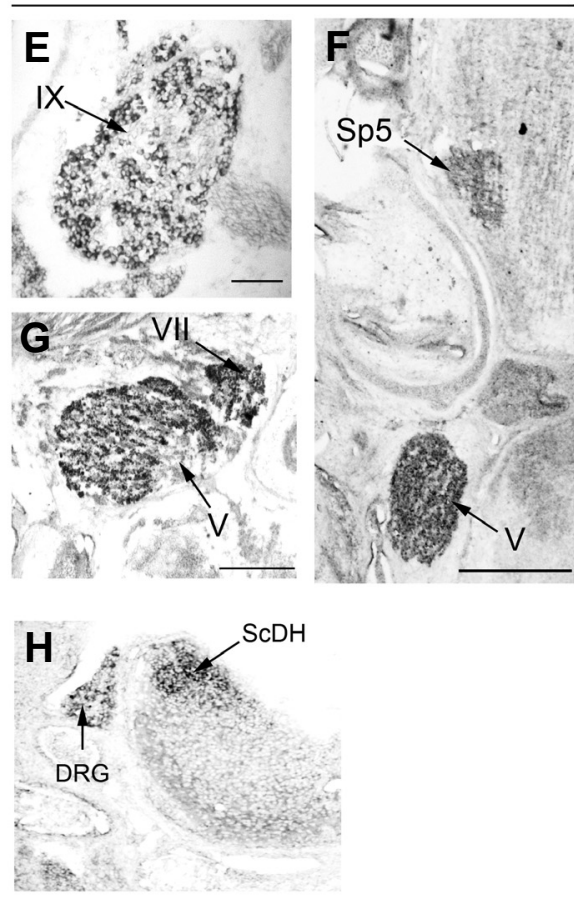

Fig. 3. Distribution of the two Prrxl1 isoforms. Comparative expression of Prrxl1 and Prrxl1-b analysed by in situ hybridization in adjacent cranial transverse sections of E15.5 embryos $(\mathbf{A}, \mathbf{B}, \mathbf{C}, \mathbf{E}, \mathbf{F}, \mathbf{G})$ and in spinal sections of $E 18.5$ embryos $(\mathbf{D}, \mathbf{H})$. V, Trigeminal ganglion; VII, Facial ganglion; IX, Glossopharingeal ganglion; Sp5, Trigeminal spinal nucleus; ScDH, Spinal cord Dorsal Horn; DRG, Dorsal root ganglion. Scale bars: $100 \mu \mathrm{m}$ in $(A, E) ; 500$ $\mu \mathrm{m}$ in $(B, C, G, F)$.

exhibited high equivalent levels of expression, whereas in the spinal cord Prrxl1-b was far less expressed than Prrxl1. These observations led us to hypothesize that Prrxl1 and Prrxl1-b could be differentially regulated in the spinal cord and DRG.

\section{Differential temporal isoform expression along devel- opment}

To further characterize putative differences in the expression pattern of Prrxl1 and Prrxl1-b, the amount of each transcript was quantified by real-time PCR analyses at different developmental ages. Although Prrxl1 expression has been previously described to be first detected at E10.5 (Rebelo et al., 2006b; 2007), this analysis started at E15.5 for spinal cord extracts and at E18.5 for DRG extracts due to difficulties in accurately dissecting these tissues at earlier ages. The time-course of expression of Prrxl1 and Prrxl1-b transcript is shown in Fig. 5A. In all the ages analyzed, the amount of Prrxl1 was higher than that of Prrxl1-b both in the DRG and the spinal cord. In the DRG, both transcripts presented the same temporal expression profile: a marked decrease of expression between E18.5 and P0 followed by an increase at P14, coming back to low levels at P21. The ratio between the two transcripts was maintained at similar values from prenatal to postnatal ages (Fig. 5B). In the spinal cord, the level of Prrxl1 mRNA declined progressively from E15.5 to P14, and then decreased abruptly to levels similar to those of Prrxl1-

b (Fig. 5A). This pattern was previously reported by western-blotting analyses (Rebelo et al., 2007), suggesting that the mRNA expression correlates with the protein expression. Prrxl1-b was expressed in high amounts at E15.5, but markedly decreased at E18.5 and maintains such low levels from then on (Fig. 5A). In contrast to what was observed in the DRG, the ratio between Prrxl1 and Prrxl1$\mathrm{b}$ in the spinal cord was high between E18.5 and postnatal day $(P) 7$, reaching a maximum value at $P 0$, and diminished progressively until P21 (Fig. 5B).

Considering the differences verified in the expression profile of the two Prrxl1 isoforms in the spinal cord (Fig. 5), we hypothesized that the precise control of the amount of Prrxl1 relative to Prrxl1-b is a key element in the regulation of the molecular mechanisms that govern the establishment of accurate sensory circuits. The importance of accurate regulation of the amount of this type of paired-like homeodomain transcription factors during development has been previously demonstrated by overexpression of an OAR truncated form of Cart1, which resulted in severe cranial and vertebral malformations due to an increase in DNA binding activity (Brouwer et al., 2003). We therefore suggest the existence of a delicate balance between Prrxl1 and its OARlacking variant, Prrxl1-b, which is critical during the nociceptive circuit development. It is known that at E15.5, an age at which the laminar architecture of the murine spinal cord begins to be perceptible, sensory axons projecting to both the superficial and deep dorsal horn have developed exhibiting a distinctive appropriate trajectory towards their central targets (Ozaki and Snider, 1997). In Prrx/1/- mice, the differentiation of superficial spinal cord layers is impaired (Chen et al., 2001), while no obvious alterations are detected in the embryonic development of DRG neurons at this age (Chen et al., 2001; Rebelo et al., 2006a). A significant reduction of the number of small primary afferent neurons was

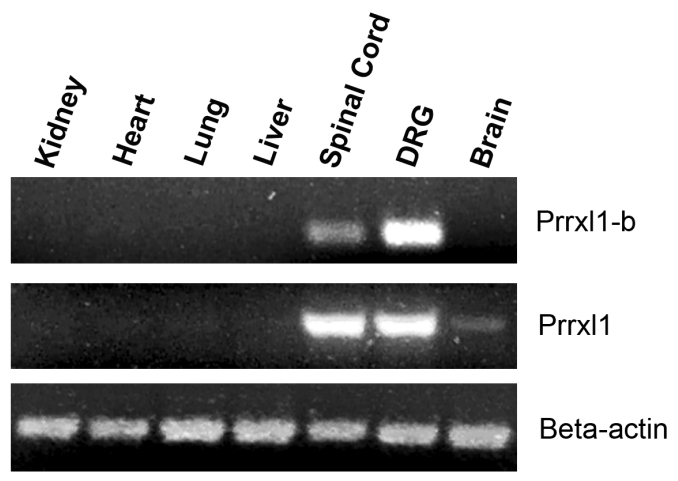

Fig. 4. Pattern of expression of the two Prrxl1 isoforms. Tissue specific expression of the Prrxl1 and Prrxl1-b analysed by reversetranscriptase PCR in E18.5 embryo. DRG, dorsal root ganglion. 
A

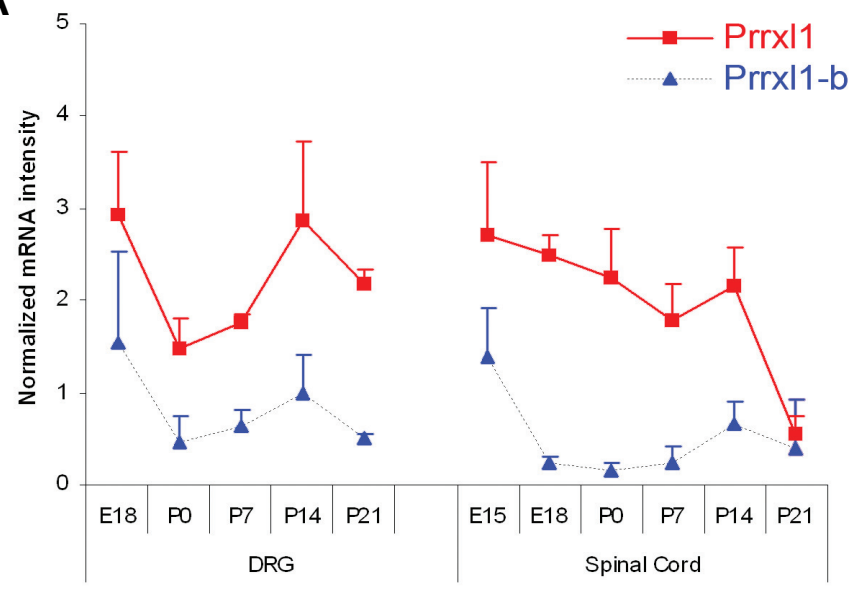

B

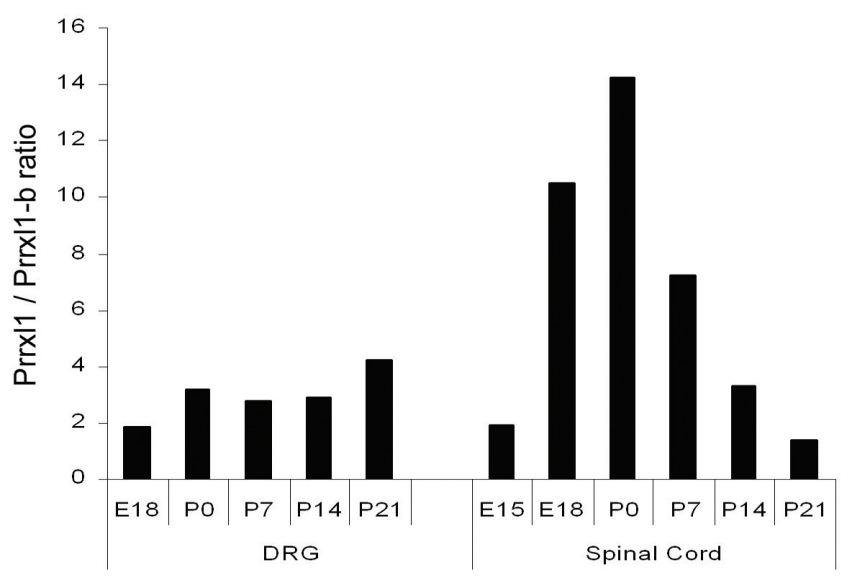

Fig. 5. Relative quantification of Prrxl1 isoform mRNA determined by real-time PCR during DRG and spinal cord development. (A) The relative $m R N A$ intensities after normalization with beta-actin at each developmental stage are represented. (B) Bars represent the ratio between Prrxl1 and Prrxl1-b mRNA intensity at the various time points.

only reported at postnatal ages (Rebelo et al., 2006a). These observations suggest that the Prrx/1 gene governs neuronal differentiation in the spinal cord and neuron survival/maintenance in the DRG. Considering the differences verified in the expression profile of the two Prrxl1 isoforms in the DRG compared to the spinal cord (Fig. 5), we hypothesize that the tissue- specific role of this gene may be sustained by accurate variations in the ratio between the amount of Prrxl1 and Prrxl1-b.

Recently, two isoforms of the Prx1 gene, another paired-like homeodomain transcription factor, have been described as opposing regulators of chondrogenesis (Peterson et al., 2005). Prx $1 a$ contains a C-terminal OAR domain while Prx $1 b$ lacks the OAR domain due to an alternatively spliced exon 4 (Norris and Kern, 2001a). The function of the conserved OAR motif is still imprecise, but deletion of this domain in the Prx1 protein leads to an increase in DNA binding and transactivation potential (Norris and Kern, 2001b). Since Prrxl1 isoforms are structurally similar to Prx1 isoforms, it is possible that Prrxl1 and Prrxl1-b are also involved in differential transcriptional activity assigned by the occurrence or not of the $\mathrm{C}$-terminal OAR motif. Taking into consid- eration the significant Prrxl1-b down-regulation observed in the spinal cord between $\mathrm{E} 18.5$ and $\mathrm{P} 7$, a time interval at which synaptic connectivity is in course (Fitzgerald, 2005), it is tempting to suggest that Prrxl1-b could be acting until that time point as a repressor of synaptic establishment at the superficial dorsal horn, modulating the Prrxl1 transcriptional activity on RGM-B, an axon guidance molecule that has been identified as a target of Prrxl1 (Samad et al., 2004). The generation of an isoform-specific Prrxl1-b genetargeted mouse is being carried out and will surely help in dissecting the specific function of this paired-like homeodomain transcription factor.

\section{Materials \& Methods}

\section{In situ hybridization}

Appropriate stage embryos, determined according to the plug date (considered to be E0.5), or dissected tissues from postnatal mice were fixed overnight with $4 \%$ paraformaldehyde buffered with $0.1 \mathrm{M}$ sodium phosphate $\mathrm{pH} 7.4$, cryoprotected with $30 \%$ sucrose, embedded in OCT compound (Sakura) and cryosectionned on $12 \mu \mathrm{m}$ sections. In situhybridization was performed to recognize Prrxl1 and Prrxl1-b mRNA following the procedures described elsewhere (Chen et al., 2001). The following primers were designed on the basis of the alternative exon 7 sequences:

Prrx/1

$5^{\prime}$-cccatgtggcatctctgaaag-3'and

$5^{\prime}$-tcatacactcttctctccctcgc-3';

\section{Prrx/1-b}

$5^{\prime}$-cccatgtggcatctctgaaag- $3^{\prime}$ and

$5^{\prime}$-tcatccttgtgtcatatctactgc-3'.

The corresponding DNA fragments were amplified by Reverse-Transcriptase PCR using E18.5 spinal cord total RNA cloned into the pCR4TOPO plasmid (Invitrogen) and sequenced to confirm the authenticity of the amplicon. After linearization, the recombinant plasmids were used as a template for the in vitroRNA transcription of digoxigenin-labeled antisense probes.

\section{Reverse-transcriptase and real-time PCR}

Total RNA from tissues of appropriate stage embryos was isolated using the Micro-to-midi total RNA purification System (Invitrogen) following the manufacturer's instructions, analyzed by typical agarose gel electrophoresis to check the RNA integrity and quantified by spectrophotometry. The first strand cDNA synthesis was prepared at $50^{\circ} \mathrm{C}$ during $1 \mathrm{~h}$ from $2 \mu \mathrm{g}$ of total RNA using $200 \mathrm{U}$ of Superscript III Reverse Transcriptase enzyme (Invitrogen) and $500 \mathrm{ng}$ of oligo(dT) ${ }_{12-18}$ (Invitrogen). The presence of Prrxl1 and its splice variant Prrxl1-b was revealed by PCR from $2 \mu \mathrm{l}$ of the first strand cDNA using the primer pairs referred above. The amount of cDNA prepared from each experimental group was normalized with an internal control by amplification of beta-actin using the primer pair 5 'tcatgaagtgtgacgttgacatcc-3' and

5 '-gtaaaacgcagctcagtaacagtc- $3^{\prime}$. The PCR conditions were the following: denaturation at $94^{\circ} \mathrm{C}$ for $30 \mathrm{~s}$, annealing at $58^{\circ} \mathrm{C}$ for $45 \mathrm{~s}$ and elongation at $72^{\circ} \mathrm{C}$ for $45 \mathrm{~s}$ during twenty-eight cycles for Prrxl1 and thirty cycles for Prrxl1-b. Equal amounts of the PCR products were subjected to a $2 \%$ agarose gel electrophoresis and visualized by ethidium bromide staining under UV light source. To assess for potential residual genomic DNA in the RNA extracts, a control containing all reagents except the reverse transcriptase enzyme was included for each sample (No RT control).

For the real-time PCR quantification, the $20 \mu \mathrm{L}$ reaction included $10 \mu \mathrm{L}$ of IQ Supermix (Bio-Rad), $400 \mathrm{nM}$ of each primer pair and $2 \mu \mathrm{L}$ of cDNA samples. RT-qPCR was performed on a iCycler iQ realtime thermocycler (Bio-Rad) using the following parameters: an initial denaturation step of 3 min at $94^{\circ} \mathrm{C}, 40$ cycles at $94^{\circ} \mathrm{C}$ for $30 \mathrm{~s}, 58^{\circ} \mathrm{C}$ for $30 \mathrm{~s}$ and $68^{\circ} \mathrm{C}$ for $30 \mathrm{~s}$. Specificity of each reaction was ascertained by melting curve analysis, which began at $50^{\circ} \mathrm{C}$ and increased to $94^{\circ} \mathrm{C}$ in $1{ }^{\circ} \mathrm{C}$ increments, and by 
agarose gel electrophoresis of the final products. To evaluate the relative PCR efficiencies of both Prrxl1 and Prrxl1-b primers, serial dilutions of first strand product were used to construct standard curves for each gene measurement. Each reaction condition was performed in duplicate and the mean values were used for calculations of mRNA expression. $\beta$-actin was used as control to confirm that similar amounts of starting cDNA were used for all stages tested. Minus Reverse Transcriptase and no RNA controls gave similar high threshold cycle values ( $>37$ cycles), demonstrating that contamination did not interfere with the quantified product. Relative fold expression and standard deviation values were calculated as per Livak and Schmittgen (2001). The results are shown as the mean \pm SE of three separate quantitative PCR from at least two independent RNA extractions.

\section{Rapid amplification of cDNA ends (RACE)}

$5^{\prime}$ and $3^{\prime}$-RACE reactions were performed using the FirstChoice RLMRACE kit (Ambion) with $1 \mu \mathrm{g}$ of total RNA extracted from mouse E15.5 spinal cord embryo as the starting material, following the instruction manual. For the $5^{\prime}$-RACE, the primers

Prrx/1

$5^{\prime}$-ccgtgcggttgctctggcag-3' (outer) and

$5^{\prime}$-gaaggagagtcccatcgggtctgg-3' (inner) and

Prrx/1-b

$5^{\prime}$-ctgttccctctgaacccaaagc-3' (outer) and

5'-ccagaacatagcattgctcggaaatg-3' (inner)

were used in the initial and nested PCR amplifications, respectively. For the 3'-RACE, the primers 5'-ctgaaagggggcccactg-3' for Prrxl1 and 5'ctgaaagatcatttccgaatg- $3^{\prime}$ for Prrxl1-b were used in a unique amplification reaction. The PCR program used was the following: $94^{\circ} \mathrm{C}$ for $1 \mathrm{~min}, 35$ cycles at $94^{\circ} \mathrm{C}$ for $30 \mathrm{~s}, 58^{\circ} \mathrm{C}$ for $30 \mathrm{~s}$ and $72^{\circ} \mathrm{C}$ for 3 min and a final extension cycle of $72^{\circ} \mathrm{C}$ for $10 \mathrm{~min}$. The amplified PCR fragments were analysed by $1.5 \%$ agarose gel electrophoresis and the higher base pairs band was extracted, cloned into the pCR2.1-TOPO vector (Invitrogen) and sequenced.

\section{Acknowledgements}

This work was funded by the FCT grant POCI/SAU-NEU/59093/2004.

\section{References}

BROUWER, A., TEN BERGE, D., WIEGERINCK, R. and MEIJLINK, F. (2003). The OAR/aristaless domain of the homeodomain protein Cart1 has an attenuating role in vivo. Mech Dev. 120: 241-252.

CHEN, Z-F., REBELO, S., WHITE, F., MALMBERG, A.B., BABA, H., LIMA, D. WOOLF, C.J., BASBAUM, A.I. and ANDERSON, D.J. (2001). The paired homeodomain protein DRG11 is required for the projection of cutaneous sensory afferent fibers to the dorsal spinal cord. Neuron. 31: 59-73.

DING, Y.Q., YIN, J., XU, H.M., JACQUIN, M.F. and CHEN, Z-F. (2003). Formation of whisker-related principal sensory nucleus-based lemniscal pathway requires a paired homeodomain transcription factor, Drg11. J. Neurosci. 23: 7246-7254.

DRAGAN, A.I., LI, Z., MAKEYEVA, E.N., MILGOTINA, E.I., LIU, Y., CRANEROBINSON, C. and PRIVALOV, P.L. (2006). Forces Driving the Binding of Homeodomains to DNA. Biochemistry. 45: 141-151.

EPPIG, J.T., BULT, C.J., KADIN, J.A., RICHARDSON, J.E., BLAKE, J.A., and the members of the Mouse Genome Database Group. (2005). The Mouse Genome Database (MGD): from genes to mice-a community resource for mouse biology. Nucleic Acids Res. 33: D471-D475.
FITZGERALD, F. (2005). The development of nociceptive circuits. Nat. Rev. Neurosci. 6: 507-520.

GAGE, P.J. and CAMPER, S.A. (1997). Pituitary homeobox 2, a novel member of the bicoid-related family of homeobox genes, is a potential regulator of anterior structure formation. Hum. Mol. Genet. 6: 457-464.

GEHRING, W.J., AFFOLTER, M. and BURGLIN, T. (1994). Homeodomain Proteins. Annual Review of Biochemistry. 63: 487-526.

GEHRING, W.J. (1992). The homeobox in perspective. Trends Biochem Sci. 8: 277-280.

LIVAK, K.J. and SCHMITTGEN, T.D. (2001). Analysis of relative gene expression data using real-time quantitative PCR and the $2^{-\Delta \Delta C}$ (-delta delta $\mathrm{c}(\mathrm{T})$ ) method. Methods 25: 402-408.

NORRIS, R.A. and KERN, M.J. (2001a). The identification of Prx1 transcription regulatory domains provides a mechanism for unequal compensation by the Prx1 and Prx2 loci. J. Biol. Chem. 276: 26829-26837.

NORRIS, R.A. and KERN, M.J. (2001b). Identification of domains mediating transcription activation, repression, and inhibition in the paired-related homeobox protein, Prx2. DNA Cell Biol. 20: 89-99.

OZAKI, S. and SNIDER, W.D. (1997). Initial trajectories of sensory axons toward laminar targets in the developing mouse spinal cord. J. Comp. Neurol. 380: 215 229.

PETERSON, R.E., HOFFMAN, S. and KERN, M.J. (2005). Opposing roles of two isoforms of the Prx1 homeobox gene in chondrogenesis. Dev. Dyn. 33: 811-821.

QIAN, Y., SHIRASAWA, S., CHEN, C., CHENG, L. and MA, Q. (2002). Proper development of relay somatic sensory neurons and D2/D4 interneurons requires homeobox genes $R n x / T / x-3$ and T/x-1. Genes Dev. 16: 1220-1233.

REBELO, S., CHEN, Z.F., ANDERSON, D.J. and LIMA, D. (2006a). Involvement of DRG11 in the development of the primary afferent nociceptive system. Mol. Cell Neurosci. 33: 236-246.

REBElo, S., REGuenGA, C., LOPES, C., PEREIRA, C., ANDERSON, D. and LIMA, D. (2006b). Cellular diversity of superficial dorsal horn glutamatergic neurons revealed by differential co-expression of Drg11, Tlx3, and Lmx1b. Program No. 717.4. Neuroscience Meeting Planner. Atlanta, GA. Society for Neuroscience.

REBELO, S., REGUENGA, C., OSÓRIO, L., PEREIRA, C., LOPES, C. and LIMA, D. (2007) DRG11 immunohistochemical expression during embryonic development in the mouse. Dev. Dyn. 236: 2653-2660.

SEMINA, E.V., REITER, R., LEYSENS, N.J., ALWARD, M., SMALL, K.W., DATSON, N.A., SIEGEL-BARTELT, J., BIERKE-NELSON, D., BITOUN, P., ZABEL, BU. CAREY, J.C. and MURRAY, J.C. (1996) Cloning and characterization of a novel bicoid-related homeobox transcription factor gene, RIEG, involved in Rieger syndrome. Nat. Genet. 14: 392-399.

SAITO, T., GREENWOOD, A., SUN, Q. and ANDERSON, D.J. (1995) Identification by differential RT-PCR of a novel paired homeodomain protein specifically expressed in sensory neurons and a subset of their CNS targets. Mol. Cell Neurosci. 6: 280-292.

SAMAD, T.A., SRINIVASAN, ’, KARCHEWSKI, L.A, JEONG, S.J., CAMPAGNA, J.A., JI, R.R., FABRIZIO, D.A., ZHANG, Y., LIN, H.Y., BELL, E. and WOOLF C.J. (2004). DRAGON: a member of the repulsive guidance molecule-related family of neuronal- and muscle-expressed membrane proteins is regulated by DRG11 and has neuronal adhesive properties. J. Neurosci. 24: 2027-2036.

TREISMAN J, HARRIS E, DESPLAN C. (1991). The paired box encodes a second DNA-binding domain in the paired homeo domain protein. Genes Dev. 5: 594604.

WILSON D, SHENG G, LECUIT T, DOSTATNI N, DESPLAN C. (1993). Cooperative dimerization of paired class homeodomains on DNA. Genes Dev. 11:21202134. 


\section{Further Related Reading, published previously in the Int. J. Dev. Biol.}

See our recent Special Issue Fertilization, in honor of David L. Garbers and edited by Paul M. Wassarman and Victor D. Vacquier at: http://www.ijdb.ehu.es/web/contents.php?vol=52\&issue=5-6

\section{Function and specificity of Hox genes}

David Foronda, Luis F. de Navas, Daniel L. Garaulet and Ernesto Sánchez-Herrero

Int. J. Dev. Biol. (2008) 52: doi: 10.1387/ijdb.072462df

The N-terminus zinc finger domain of Xenopus SIP1 is important for neural induction, but not for suppression of Xbra expression

Kazuhiro R. Nitta, Shuji Takahashi, Yoshikazu Haramoto, Masakazu Fukuda, Kousuke

Tanegashima, Yasuko Onuma

and Makoto Asashima

Int. J. Dev. Biol. (2007) 51: 321-325

The expression and alternative splicing of alpha-neurexins during Xenopus development

Zhihong Zeng, Colin R. Sharpe, J. Paul Simons and Dariusz C. Górecki

Int. J. Dev. Biol. (2006) 50: 39-46

Regulation of gene expression by Pax6 in ocular cells: a case of tissue-preferred expression of crystallins in lens

Ales Cvekl, Ying Yang, Bharesh K. Chauhan and Kveta Cveklova

Int. J. Dev. Biol. (2004) 48: 829-844

Isolation and growth factor inducibility of the Xenopus laevis Lmx1b gene

Caroline E Haldin, Sarjit Nijjar, Karine Massé, Mark W Barnett and Elizabeth A Jones

Int. J. Dev. Biol. (2003) 47: 253-262

5 yr ISI Impact Factor $(2008)=3.271$

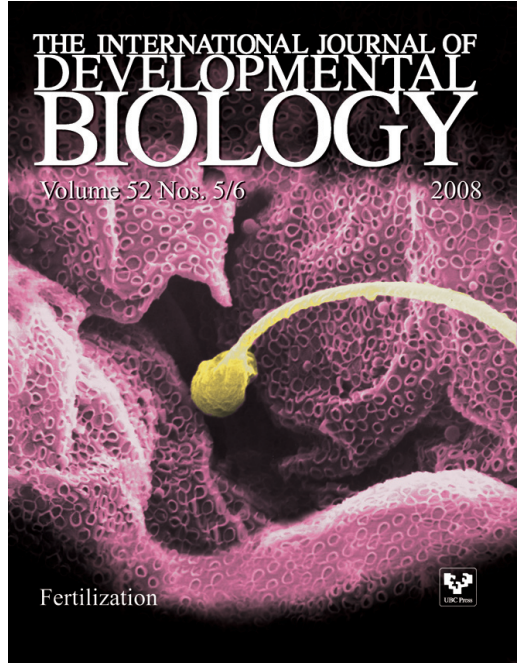

